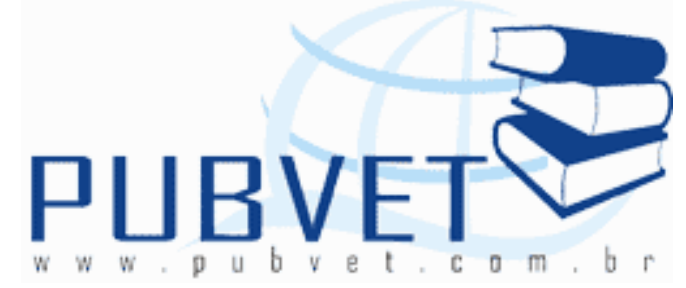

PUBVET, Publicações em Medicina Veterinária e Zootecnia.

\title{
Variação regional da perda de calor latente na superfície corporal de ovinos Morada Nova em uma região semiárida
}

João Batista Freire de Souza Junior, Hérica Girlane Tertulino Domingos, Maiko Roberto Tavares Dantas, Janio Lopes Torquato, Geovan Figueirêdo Sá Filho, Leonardo Lelis de Macedo Costa

Universidade Federal Rural do Semi-Árido, Departamento de Ciências Animais, Laboratório de Biometeorologia, Biofísica Ambiental e Bem-Estar Animal (LABBEA), BR 110, Km 47, Costa e Silva, CEP: 59625-900, Mossoró-RN.

\section{Resumo}

O objetivo do presente estudo foi avaliar a perda de calor latente em diferentes regiões da superfície corporal de ovinos na região semiárida do Brasil. Foram utilizados 4 ovinos da raça Morada Nova com idade entre 12 e 18 meses. Os animais permaneceram à sombra de um aprisco coberto por telhas cerâmicas e com pé-direito de 3 metros. Foram aferidas a temperatura do ar, a umidade relativa do ar, velocidade do ar, a temperatura do globo, a evaporação cutânea, a temperatura de superfície do pelame dos animais e o fluxo de calor latente na superfície cutânea dos animais, para realização dos cálculos. Os resultados da análise de variância mostraram que o efeito região corporal foi significativo $(P=0,0256)$. A média mais elevada da perda de calor latente na superfície cutânea $(E S)$ foi aferida no pescoço $\left(123,54 \mathrm{~W} \mathrm{~m}^{-2}\right.$ ), a qual diferiu significativamente $(P<0,05)$ da média encontrada na coxa. No 
entanto, esta média encontrada no pescoço não diferiu da média do flanco $\left(88,65 \mathrm{~W} \mathrm{~m}^{-2}\right)$, onde, esta última, não diferiu da média encontrada na coxa $\left(69,47 \mathrm{~W} \mathrm{~m}^{-2}\right)$. A perda de calor latente não é distribuída de forma homogênea na superfície corporal de ovinos da raça Morada Nova em ambiente semiárido.

Palavras-chave: ovinos, resfriamento evaporative, superfície corporal, termorregulação

\title{
Regional variation of the latent heat loss in the body surface of sheep in a semiarid region
}

\begin{abstract}
The aim of the present study was to evaluate the latent heat loss in different regions of the body surface of sheep in the semiarid region of Brazil. We used four Morada Nova sheeps between 12 to 18 months old. The animals remained in the shadow of a sheepfold covered with ceramic tiles and ceiling height of 3 meters. We measured the air temperature, relative humidity, wind speed, temperature of the globe, cutaneous evaporation, the surface temperature of the fur of animals and latent heat flux on the cutaneous surface of animals, to perform the calculations. The results of analysis of variance showed that the effect of body region was significant $(P=0.0256)$. The highest average in loss of latent heat of the cutaneous surface (Es) was measured in the neck (123.54 $\mathrm{W} \mathrm{\textrm {m } ^ { - 2 }}$ ), which differed significantly $(\mathrm{P}<0.05)$ of the average found in the thigh. However, this average found in the neck did not differ from average of the flank $\left(88.65 \mathrm{~W} \mathrm{~m}^{-2}\right)$, where, this last, did not differ from the average found in the thigh $\left(69.47 \mathrm{~W} \mathrm{~m}^{-2}\right)$. The latent heat loss is not distributed homogeneously throughout the body surface of Morada Nova sheeps in semiarid environment.
\end{abstract}

Keywords: sheep, evaporative cooling, body surface, thermoregulation 


\section{INTRODUÇÃO}

O resfriamento por evaporação cutânea é um importante meio de controle térmico proporcionado pela sudação, ocorrendo em ovinos principalmente no nível da epiderme, respondendo por, aproximadamente, 80\% da perda total (MCLEAN 1963; SILVA \& STALING, 2003; SILVA \& MAIA et al., 2011). No entanto, essa evaporação não é homogeneamente distribuída sobre a superfície corporal (SOUZA JR, 2009), o mesmo ocorrendo com a temperatura da superfície do pelame (MAIA et al., 2005).

Assim, o objetivo deste trabalho foi avaliar a perda de calor latente em diferentes regiões da superfície corporal de ovinos na região semiárida do Brasil.

\section{MATERIAL E MÉTODOS}

O trabalho foi desenvolvido no Setor Produtivo de Pequenos Ruminantes da Universidade Federal Rural do Semi-Árido (UFERSA), localizado em Mossoró/RN (latitude $05^{\circ} 11^{\prime}$ S e longitude $37^{\circ} 22^{\prime} \mathrm{W}$ ). Foram utilizados 4 ovinos da raça Morada Nova com idade entre 12 e 18 meses. Os animais permaneceram à sombra de um aprisco coberto por telhas cerâmicas e com pé-direito de 3 metros. A temperatura do $\operatorname{ar}\left(T_{A},{ }^{\circ} \mathrm{C}\right)$ e a umidade relativa do ar $\left(U_{R}, \%\right)$, foram aferidas com um termo-higrômetro digital modelo HT-300 (INSTRUTHERM). A temperatura do globo $\left(T_{G},{ }^{\circ} \mathrm{C}\right)$ foi aferida utilizando um sensor de temperatura introduzido em um globo negro (esfera oca de chapa de cobre, com 0,15 $\mathrm{m}$ de diâmetro e pintada de preto fosco). A velocidade do vento $\left(V_{V}, \mathrm{~m} \mathrm{~s}^{-1}\right)$ foi aferida com um termo-anemômetro de precisão (Alnor APM-360).

A evaporação cutânea foi medida em regiões da pele selecionadas de área $0,00724 \mathrm{~m}^{2}$ cada, por meio de uma cápsula ventilada similar a 
desenvolvida por Maia et al. (2005). As medidas foram feitas em três regiões do corpo (pescoço, flanco e coxa), onde a cápsula ventilada foi utilizada manualmente na posição durante o tempo necessário para cada leitura.

A evaporação cutânea foi o produto do fluxo de ar através da cápsula $\left(f_{A C}, \mathrm{~m}^{3} \mathrm{~s}^{-1}\right)$ pela diferença na umidade absoluta do ar entrando $\left(\psi_{A}, \mathrm{~g} \mathrm{~m}^{-3}\right)$ e saindo $\left(\psi_{A C}, \mathrm{~g} \mathrm{~m}^{-3}\right)$ da cápsula, sendo esse produto dividido pela área da seção da cápsula $\left(A_{C}=0,00724 \mathrm{~m}^{2}\right)$. O fluxo de ar através da cápsula foi obtido pelo produto da área da seção do tubo de saída de ar da cápsula (que tem $0,010749 \mathrm{~m}$ de raio) pela velocidade do ar passando no interior da cápsula sobre a área de teste $\left(V_{A C}, \mathrm{~m} \mathrm{~s}^{-1}\right)$. A velocidade e a temperatura $\left(T_{A C}, \mathrm{~K}\right)$ do ar passando no interior da cápsula sobre a área de teste foram medidas com a ponta de prova de um termo-anemômetro de precisão (Alnor APM-360) inserido no tubo de saída da cápsula $C$. A perda de calor latente na superfície cutânea $\left(E_{S}, W \mathrm{~m}^{-2}\right)$ foi estimada como sendo o produto da evaporação cutânea $\left(E_{C}, \mathrm{~g} \mathrm{~s}^{-1} \mathrm{~m}^{-2}\right)$ pelo calor latente de vaporização da água: $\mathrm{E}_{\mathrm{S}}=\lambda E_{C}$

A análise de variância foi realizada pelo método dos quadrados mínimos e as médias foram comparadas pelo teste de Tukey-Kramer $(p<0,05)$ utilizando o programa SAS (SAS, 2001).

\section{RESULTADOS E DISCUSSÃO}

As médias gerais das variáveis meteorológicas observadas durante as aferições da perda de calor latente na superfície corporal de ovinos em uma região semiárida estão apresentadas na tabela 1 . Os resultados da análise de variância mostraram que o efeito região corporal foi significativo $(P=0,0256)$. 
Tabelas 1- Médias gerais com seus respectivos erros padrão e valores máximos e mínimos das variáveis meteorológicas aferidas durante o estudo.

\begin{tabular}{lcccc}
\hline $\begin{array}{l}\text { Variáveis } \\
\text { meteorológicas }\end{array}$ & $\mathbf{n}$ & Média & Máximo & Mínimo \\
\hline$T_{A}\left({ }^{\circ} \mathrm{C}\right)$ & 55 & $30,34 \pm 0,43$ & 34,70 & 26,40 \\
$U_{R}(\%)$ & 55 & $66,32 \pm 1,56$ & 81,30 & 51,20 \\
$T_{G}\left({ }^{\circ} \mathrm{C}\right)$ & 55 & $32,00 \pm 0,28$ & 36,10 & 26,80 \\
$V_{V}\left(\mathrm{~m} \mathrm{~s}^{-1}\right)$ & 55 & $2,25 \pm 0,05$ & 5,20 & 0,10 \\
\hline
\end{tabular}

A média mais elevada na perda de calor latente de superfície cutânea (Es) foi aferida no pescoço, a qual diferiu significativamente $(P<0,05)$ da média encontrada na coxa (Figura 1). No entanto, esta média encontrada no pescoço não diferiu da média do flanco, onde, esta última, não diferiu da média encontrada na coxa. Souza Jr (2009) estudando vacas Holandesas à sombra não encontraram diferenças significativas entre as regiões corporais, no entanto quando os animais foram expostos ao sol a $E_{S}$ medida no pescoço diferiu estatisticamente das médias de $E_{S}$ medidas no flanco e na coxa que, entretanto, não diferiram entre si, isso mostra o grande efeito da radiação solar direta que elevou acentuadamente a $T_{S}$ fazendo com que acelerasse 0 processo evaporativo na superfície corporal. Scharf et al. (2009) também encontrou diferenças na $E_{S}$ aferida em diferentes regiões da superfície corporal. 
SOUZA JÚNIOR, J.B.F. et al. Variação regional da perda de calor latente na superfície corporal de ovinos Morada Nova em uma região semiárida. PUBVET, Londrina, V. 6, N. 21, Ed. 208, Art. 1389, 2012.

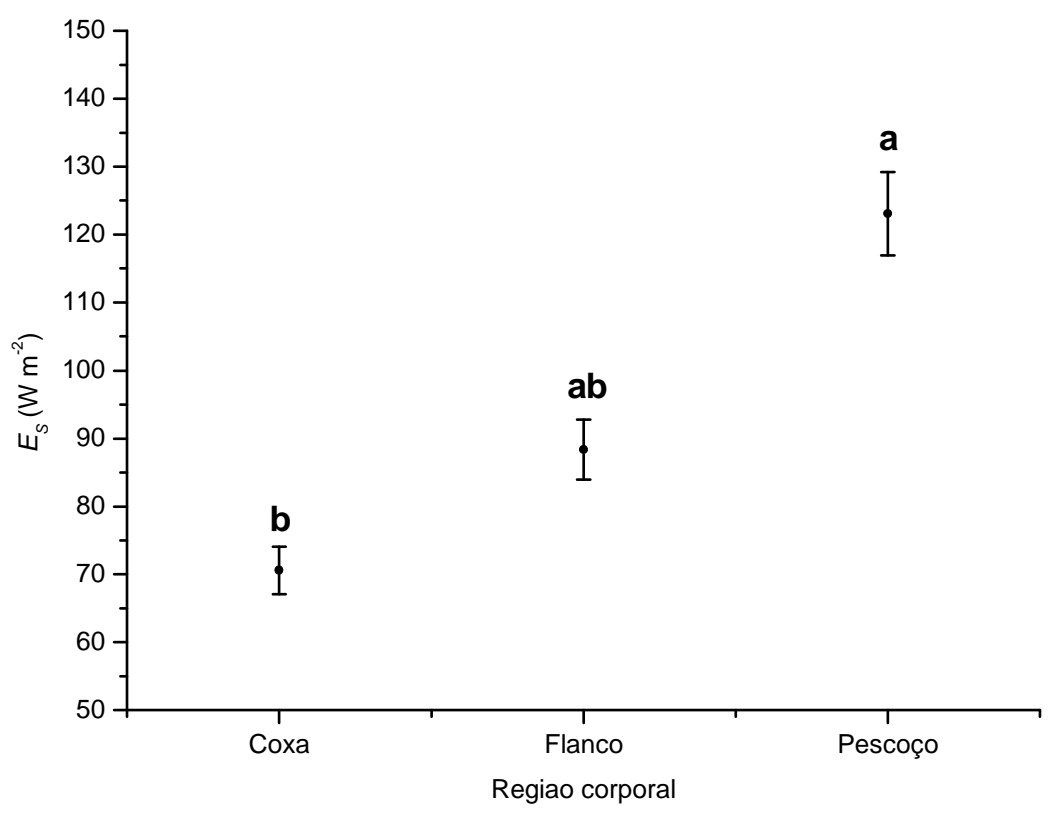

Figura 1 - Médias estimadas por quadrados mínimos da perda de calor latente nas diferentes regiões corporais estudadas. Letras minúsculas iguais, não diferem significativamente pelo teste de Tukey-Kramer $(P<0,05)$.

\section{CONCLUSÕES}

A perda de calor latente não é distribuída de forma homogênea na superfície corporal de ovinos da raça Morada Nova em ambiente semiárido. A região do pescoço contribui com a maior parte da dissipação de calor por evaporação cutânea.

\section{REFERÊNCIAS}

MAIA, A.S.C.; SILVA, R.G; BATTISTON, C.M. Sensible and latent heat loss from body surface of Holstein cows in a tropical environment. International Journal of Biometeorology, $v$. 50, p. 17-22, 2005.

MCLEAN, J.A. Measurement of cutaneous moisture vaporization from cattle by ventilated capsules. Journal of Physiology, v. 167, p. 417-426, 1963. 
SILVA, R.G.; STARLING, J.M.C. Evaporação cutânea e respiratória em ovinos sob altas temperaturas ambientes. Revista Brasileira de Zootecnia, v.32, n.6, p.1956-1961, 2003 (supl. 2).

SILVA, R. G.; MAIA, A. S. C. Evaporative cooling and cutaneous surface temperature of Holstein cows in tropical conditions. Revista Brasileira de Zootecnia, v.40, n.5, p.1143$1147,2011$.

SOUZA JR, J. B. F. Estudo da perda de calor por evaporação na superfície corporal em vacas holandesas manejadas no Semi-árido. 2009. 49f. Monografia (Graduação em Zootecnia) - Universidade Federal Rural do Semi-Árido, Mossoró-RN.

SCHARF, B.; WAX, L. E.; AIKEN, G. E.; SPIERS, D. E. Regional differences in sweat rate response of steers to short-term heat stress. Int. J. Biometeorol. v. 52, p. 725-732, 2009. 